\title{
Study Of The Influence Of Nozzle Shape On The Primary Atomization Process By Means Of Direct Numerical Simulation
}

\author{
F.J. Salvador ${ }^{1}$, J. Gimeno ${ }^{1}$, M. Carreres ${ }^{1}$, L.A. Gonzalez-Montero*1 \\ ${ }^{1}$ CMT - Motores Térmicos, Universitat Politècnica de València, Valencia 46022, España \\ ${ }^{*}$ Corresponding author email: lugonmon@mot.upv.es
}

\begin{abstract}
The atomization process is a physical phenomenon that takes place as an essential mechanism in many industrial fields. Even though it has been widely addressed in the literature, there are still several aspects of primary atomization that are far from being understood. In order to shed light on the physical processes that take place in the near-field of the injection, Direct Numerical Simulations (DNS) are rising as a fundamental tool to provide detailed data where the optical access is difficult and expensive. Nevertheless, most real engineering conditions are too complex to be accurately resolved with DNS. Hence, a more fundamental standpoint is chosen to first understand the fundamental mechanisms that drive the process. In this context, this work presents a study of the influence of the nozzle shape on droplet formation by using DNS simulations performed with the Paris-Simulator code. The injection inflow conditions are obtained by mapping results from Large Eddy Simulation (LES) of an elliptic duct with eccentricity factor of 0.85 , maintaining the bulk velocity and the crosssection area. The Reynolds number for all cases is maintained around 5000 and the Weber number around 26000. Results are compared to a round jet DNS simulation also performed with a mapped boundary condition. The main aim of the work is to understand how the elliptic cross-section eccentricity affects the atomization regime, breakup mechanism, droplet azimuthal distribution and turbulent structures.
\end{abstract}

\section{Keywords}

Droplet analysis; Direct Numerical Simulation; Elliptical jet.

\section{Introduction}

The standards applied to combustion engine emissions become stricter. Hence, the study of the atomization process is getting more attention to produce better droplet formation and thus, cleaner combustion processes. Even though this phenomenon is ubiquitous in engineering applications, many aspects of this process remain still unknown.

One of the solutions to improve liquid atomization that is getting attention is using elliptical nozzles instead of the commonly used round nozzles. This solution has been mostly addressed by experimental works: decades ago, Husain et al. [1], [2] and Ho et al.[3] studied from a theoretical approach the experiments of an elliptical jet. Recently, the comparison between round and elliptical jets using real Diesel injectors has been used as an indicator of the improvements on this new approach. In this regard, Lee et al. [4] performed a study with 2 different ellipse eccentricities and found an improvement on the spreading angle, in particular on the minor axis plane and similar results were obtained by $\mathrm{Yu}$, et al. [5].

From the computational point of view, different approaches have been made also by Yu et al. [6] performed Large Eddy Simulations (LES) with Volume-Of-Fluid (VOF) method of a singlehole elliptical nozzle. Salvador et al. [7] used the $\Sigma-Y$ model and Homogeneous Relaxation Model to evaluate the cavitation in a multi-hole elliptical geometry with different eccentricities. However, experimental and computational studies performed on past years are mainly focused far from the nozzle exit. There is no reliable information of the primary atomization of the elliptical jet in the near-field. The reason is that, in the so called 'dense region', the optical 
techniques capable to get the mass concentration or detecting droplets are scarce, complex, and very expensive; whereas the common CFD approaches model too much energy to have a proper turbulent resolution, and thus, droplet generation discretization.

Here is where Direct Numerical Simulations (DNS) are getting more attention by being able to simulate the breakup process with high fidelity. Even so, this computational technique is also difficult to be performed with real physical accuracy. When done, a deep study must be performed in order to extract the most important data to be given to experimentalists and computational researchers in the field.

In this context, the aim of the work is to study the effect of the elliptical nozzle on the droplet generation at the near-field. To this end, a round jet simulation already addressed in [8] is used as reference to study the impact of the nozzle eccentricity on the droplet distribution. The physical parameters (round nozzle diameter, discharge pressure...) are set according to the Spray A conditions from ECN [9] and the liquid properties correspond to a n-Dodecane fuel. It is worth mentioning that, even though the conditions of Spray A relate to transcritical or supercritical behaviour for $n$-dodecane, such particular effects have not been considered within the bounds of this work. For the elliptical nozzle, the parameters maintained are the injection area, bulk velocity, liquid properties, and discharge conditions, modifying the major and minor axis to achieve an eccentricity factor of 0.85 .

The paper is organized as follows: the first section introduces the algorithms used to perform the simulations, next the different cases of study are exposed, and a brief explanation of the inflow boundary conditions used. Then the results of both cases are compared, focusing on the droplet formation and distribution. Finally, the main conclusions and future research are presented.

\section{Methodology}

\section{Governing equations and numerical methodology}

The primary atomization simulations presented in this paper have been performed with the PARIS-Simulator code [10]. This code is widely used to run DNS of multiphase flows, resolving the equations of an incompressible flow on a cartesian grid:

$$
\begin{aligned}
& \nabla \cdot \boldsymbol{u}=0 \\
& \rho\left(\partial_{t} \boldsymbol{u}+\boldsymbol{u} \cdot \nabla \boldsymbol{u}\right)=-\nabla p+\nabla \cdot(2 \mu \boldsymbol{D})+\sigma \kappa \partial_{s} \boldsymbol{n}
\end{aligned}
$$

where $\boldsymbol{u}$ is the velocity field, $\rho$ is the fluid density, $p$ is the pressure field, $\mu$ is the fluid dynamic viscosity and $\boldsymbol{D}$ is the deformation tensor described as $\boldsymbol{D}=\left(\partial_{i} u_{j}+\partial_{j} u_{i}\right) / 2$. Last term of Eq. 2 accounts for the surface tension stressed, being $\sigma$ the surface tension, $\kappa$ the curvature of the liquid surface, $\partial_{s}$ a Dirac distribution that concentrates the effect of this source term on the fluid surface and $\boldsymbol{n}$ the normal direction of the liquid surface.

PARIS uses the VOF method to model the multiphase nature of the flow. This method uses an advection equation for the volume fraction $C$ :

$$
\partial_{t} C+\boldsymbol{u} \cdot \nabla C=0
$$

So, the density and viscosity can be computed as the arithmetic mean:

$$
\begin{aligned}
& \rho=C \rho_{l}+(1-C) \rho_{g} \\
& \mu=C \mu_{l}+(1-C) \mu_{g}
\end{aligned}
$$

where $l$ and $g$ subscripts represent the liquid and gas phase properties, respectively. This code implements many ideas from [11]. 
As already mentioned, the main objective of this work is the study of the nozzle shape influence on the primary atomization, focusing specifically on elliptic cross-section eccentricity. For this purpose, a round jet simulation with operating conditions resembling the Spray $A$ configuration (nozzle diameter, discharge pressure ...) has been chosen as a reference. Table 1 shows the physical properties used for both simulations, whereas Table 2 gathers the simulation parameters used. It is worth mentioning that although the injection area, liquid properties and spray mean velocity are unchanged for the elliptical jet, the Reynolds is slightly lower because it is computed with the hydraulic diameter ( $84.30 \mu \mathrm{m}$ for this shape).

Table 1 - Physical properties

\begin{tabular}{c|c}
\hline & Values \\
\hline Spray mean velocity & $100 \mathrm{~m} / \mathrm{s}$ \\
Fuel density & $750 \mathrm{~kg} / \mathrm{m}^{3}$ \\
Fuel viscosity & $1.34 \cdot 10^{-3} \mathrm{~Pa} \cdot \mathrm{s}$ \\
Fuel/Nitrogen surface tension & $2.535 \cdot 10^{-5} \mathrm{~N} / \mathrm{m}$ \\
Nitrogen density & $22.8 \mathrm{~kg} / \mathrm{m}^{3}$ \\
Nitrogen viscosity & $1.85 \cdot 10^{-5} \mathrm{~Pa} \cdot \mathrm{s}$
\end{tabular}

Table 2 - Simulation parameters

\begin{tabular}{c|c|c}
\hline & Round jet & Elliptic jet \\
\hline Eccentricity & 0 & 0.85 \\
Reynolds Number & 5037 & 4718 \\
Major axis [ $\mu \mathrm{m}]$ & 90 & 126.65 \\
Minor axis $[\mu \mathrm{m}]$ & 90 & 65.63 \\
Cell size $[\mu \mathrm{m}]$ & 2.343 & 2.343 \\
x - length [mm] & 2.4 & 2.4 \\
y/z - length [mm] & $1.2 \times 1.2$ & $1.8 \times 1.2$ \\
Timestep [ns] & 4 & 4 \\
Simulation time [ms] & 300 & 300
\end{tabular}

The cell size used has been tested on the round jet, giving a cell size-Kolmogorov scale ratio of 5 , which practically resolves the total of the energy [8]. Hence, even though the turbulence study of the elliptical case is still to be performed, we expect good turbulence resolution.

\section{Inflow boundary condition}

One of the most important aspects of performing DNS simulations is imposing coherent boundary conditions that will trigger the turbulence, and thus the atomization, within the computational domain. In order to generate reliable boundary conditions, different LES have been performed in pipe domains whose cross-section match the nozzle shape and the same physical properties as for the DNS simulations (Table 1). These simulations have been performed using OpenFOAM [12] with the pisoFOAM solver. In order to reach a statistically steady solution, a periodic boundary condition is applied to the inlet and outlet boundary condition. Snapshots of the axial velocity field are depicted in Figure 1.

Turbulent statistics are checked once the steady state is reached in order to validate the results of the mean velocity profile and the velocity deviation for both BCs. Finally, once the LES are validated, the results are interpolated into a Cartesian mesh and fed to DNS. 


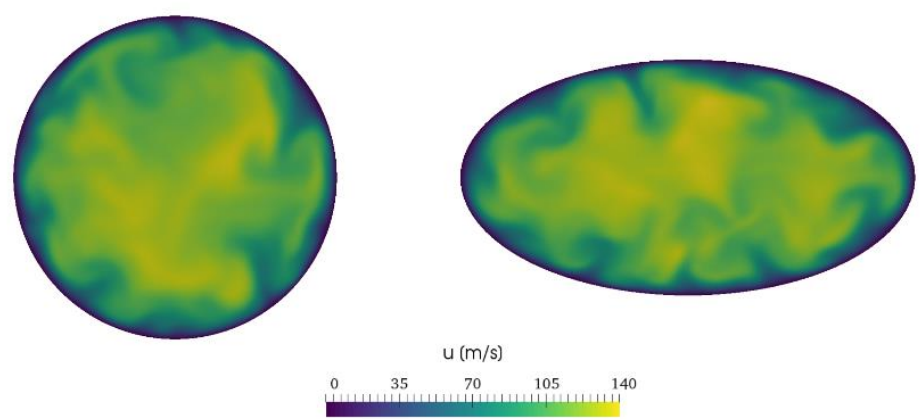

Figure 1: velocity field snapshot of a cross-section from left, round nozzle and right, elliptical nozzle.

\section{Droplet detection algorithm}

The main efforts of this study are focused on the granulometric analysis of the droplet cloud generated from both sprays. The whole domain is analyzed looking for all the liquid structures detached from the spray liquid core. In this work, a continuous liquid structure defined by free surfaces is considered as a droplet. The whole domain is recursively scanned so that every droplet is identified.

Droplet statistics such as the velocity components are averages of the velocity components on each cell composing the droplet and are placed at the center of mass of the liquid structure. This methodology has already proven its capabilities in previous studies [13], [14].

From the experimental point of view, the droplets defined in this manner correspond to liquid structures that do not appear in optical connectivity analyses. Regarding the computational approach, these results can be useful to improve a Discrete Droplet Model (DDM).

Finally, this algorithm is applied every $0.5 \mu s$ during the transient stage (until the spray tip reaches the end of the axial domain), and then every $10 \mu s$ until the end of the simulation time.

\section{Results and Discussion}

Figure 2 shows a snapshot of the contour of the iso-colour function $C$ for both cases, coloured with the velocity. These snapshots have been taken at $t=60 \mu \mathrm{s}$, when the spray tip has reached the end of the axial domain. The upper figures correspond to the round spray, whereas the bottom ones correspond to the elliptical spray. Left figures represent an axial overview of the spray development, whereas the right figures show a cross-section located at $2 \mathrm{~mm}$ from the nozzle outlet. When looking at the overall spray figures, the elliptical spray shows a shorter non-disturbed length, parameter associated to a higher atomization rate. On the other hand, the clipped view depicts a similar behaviour for both cases. However, this is a single snapshot, and this first qualitative morphological study needs to be complemented with a deeper granulometry analysis.

As a first approach to the droplet generation, the total number of droplets detected is processed for the whole simulation time. Figure 2 depicts the time evolution of the number of droplets detected for the two cases. The behaviour of this parameter is similar in both cases, exhibiting a steep slope when the spray is penetrating into the domain increasing the droplet generation rate. Once the spray tip leaves the domain, the number of droplets tends to stabilize. When comparing both cases, the elliptical nozzle generates more droplets than the round nozzle. Averaging the number of droplets detected when $t>60 \mu \mathrm{s}$, the elliptical nozzle produces nearly $20 \%$ more droplets than the reference case. 


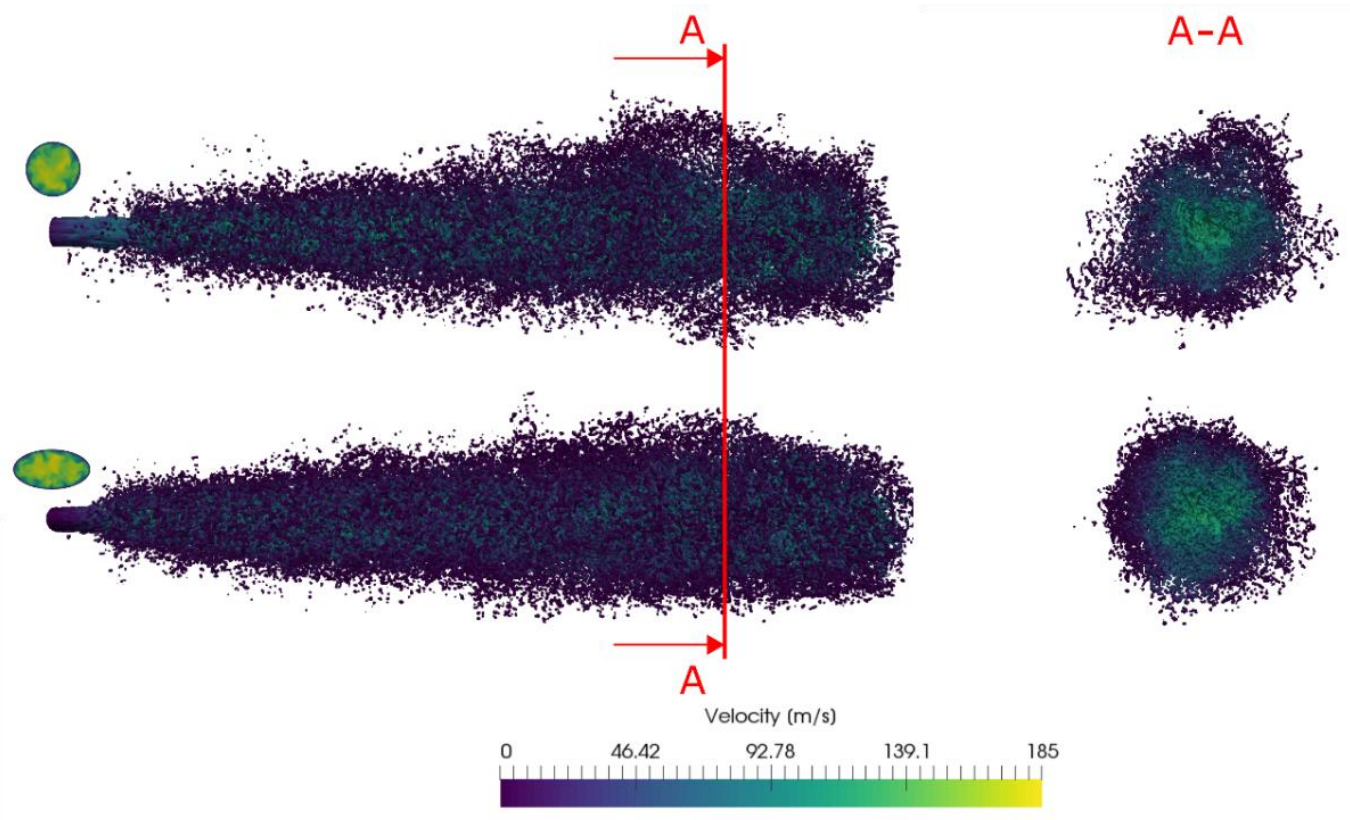

Figure 2: Snapshot for $t=60 \mu \mathrm{s}$ of the spray morphology. Up: the round spray case, bot: the elliptical spray case. Left: axial spray morphology, right: transversal clip at $2 \mathrm{~mm}$ from the nozzle

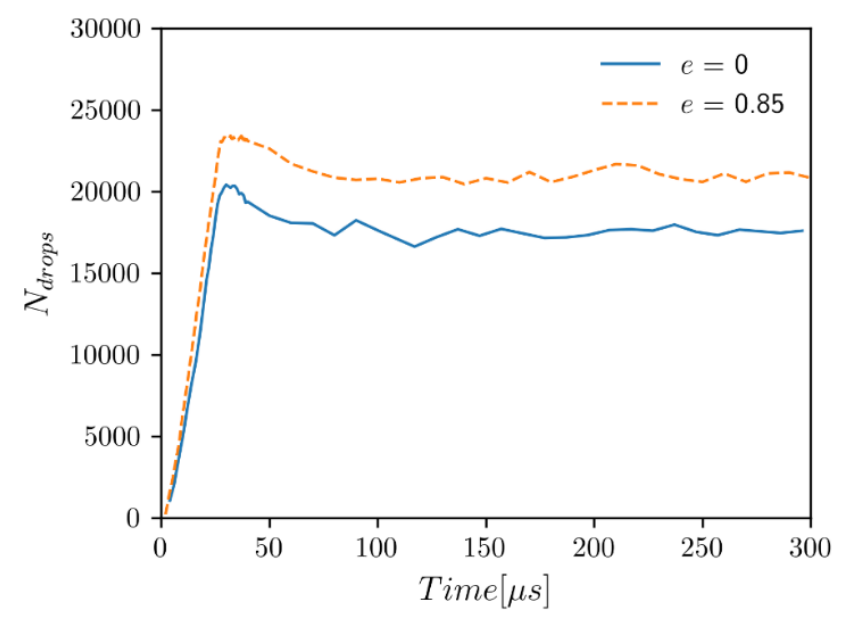

Figure 3: Time evolution of the number of droplets detected.

When it comes to the atomization study, aside from the total number of droplets generated, the size distribution of the droplet cloud is of fundamental significance. A finer droplet generation leads to a better evaporation and thus, a better combustion. Hence, a volumetric diameter is calculated for each droplet using its liquid volume as:

$$
d_{v}=\sqrt[3]{6 \cdot \frac{V}{\pi}}
$$

Then, all droplets are sorted according to this parameter in order to get a droplet size distribution. Figure 4 left shows the averaged droplet size distribution for both cases. The averaging is performed starting when the spray tip reaches the end of the axial domain until the end of the simulation time. Here, the elliptical nozzle shows an increase of small droplets with respect to the round nozzle, but a decrease on the bigger droplets. It is worth mentioning that droplets with $d_{v}$ around $25 \%$ of the round nozzle diameter are more probably to be liquid 
ligaments detached from the spray core due to turbulent shear stresses, whereas the smaller ones come from ligament breakup. Also, the values of $d_{v}$ obtained are similar to the droplet diameter measured on the Argonne National Lab [15]. This trend is also visible when applying the Probability Density Function (PDF) to the droplet size distribution. As Figure 4 right shows, the maximum peak is located at the same position which corresponds to a droplet diameter twice the cell size. This size is the minimum size for which a droplet can be considered as a 'real' droplet because the code is able to resolve it. However, for diameters greater than $7.5 \mu \mathrm{m}$ the round jet starts having higher PDF. This means that is more probable to have smaller droplets in the elliptical case than the in the round nozzle.
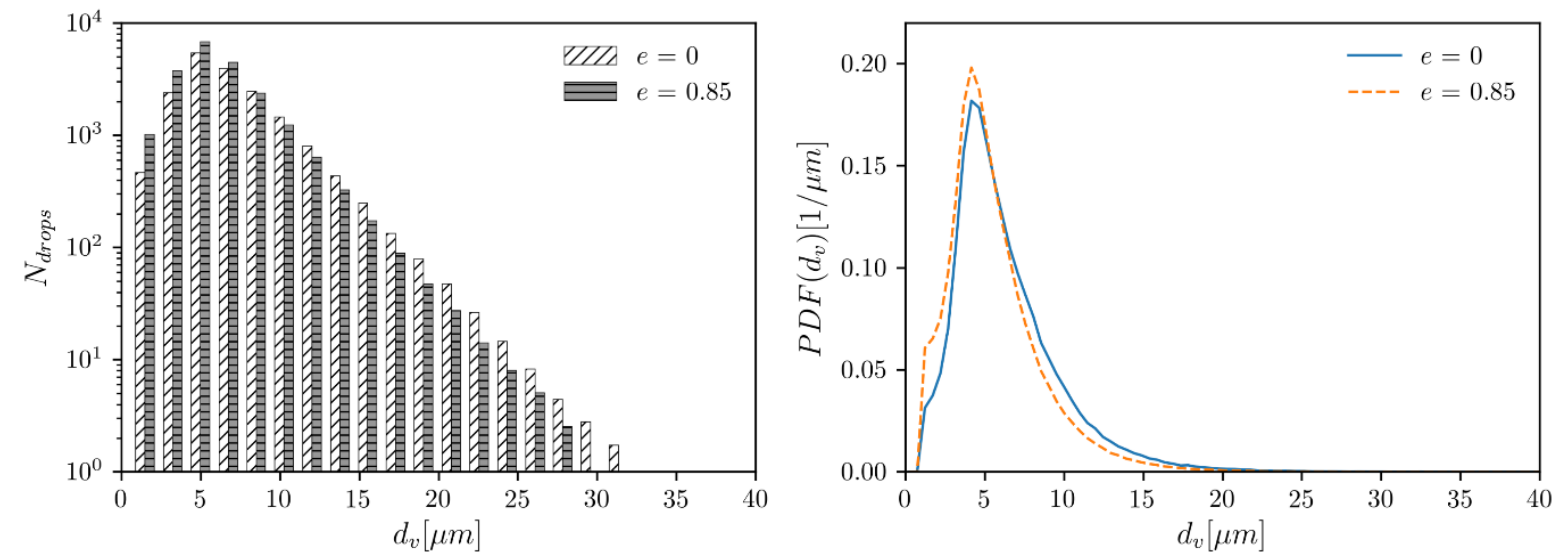

Figure 4: Left: time-averaged number of droplets sorted by volumetric diameter, right: time-averaged Probability Density Function of the droplet size.

Apart from the droplet size, it is interesting to study how they are distributed over the domain in order to check if the spray opening angle is influenced by the nozzle shape. Being that the elliptical jet is not showing an axisymmetric behaviour as the round jet, a 2D probability function perpendicular to the axial direction is performed. The cross-section is divided in squared parcels, so that the number of cells located in each parcel divided by the total amount of cells gives the probability of finding a droplet in that position. This bi-dimensional probability function has been computed with the droplets at an axial position over $2 \mathrm{~mm}$ from the nozzle in order to study the most developed region of the spray. Figure 5 shows the comparison between both cases collapsing all data in one quadrant. As expected, the round jet shows an axisymmetric pattern while the elliptical spray shows an elliptical pattern. Although the minor axis from the elliptical nozzle is around $28 \%$ shorter than the radius of the round one, it presents a similar spray aperture than the round jet. On the other hand, the major axis is $40 \%$ bigger than the radius, but the aperture is only slightly bigger, which means that the spray angle on the minor axis plane is wider than the one detected on the major axis plane. This trend is already reported in the literature from both experimental [5] and computational [6] works, where different configurations were simulated and the spray aperture for both major and minor axis tend to collapse to the same angle within $1-5 \mathrm{~mm}$.

The results presented in this work show an increase of droplet generation for the elliptical nozzle for nearly the same injection condition, as well a wider spray aperture and a trend of shifting into a circular shape once the spray is developed. On the other hand, the biggest unknown would be the how turbulent processes that take place in the elliptical nozzle evolve during the atomization domain, leading to this behaviour and also, the temporal evolution of the generated droplets. 


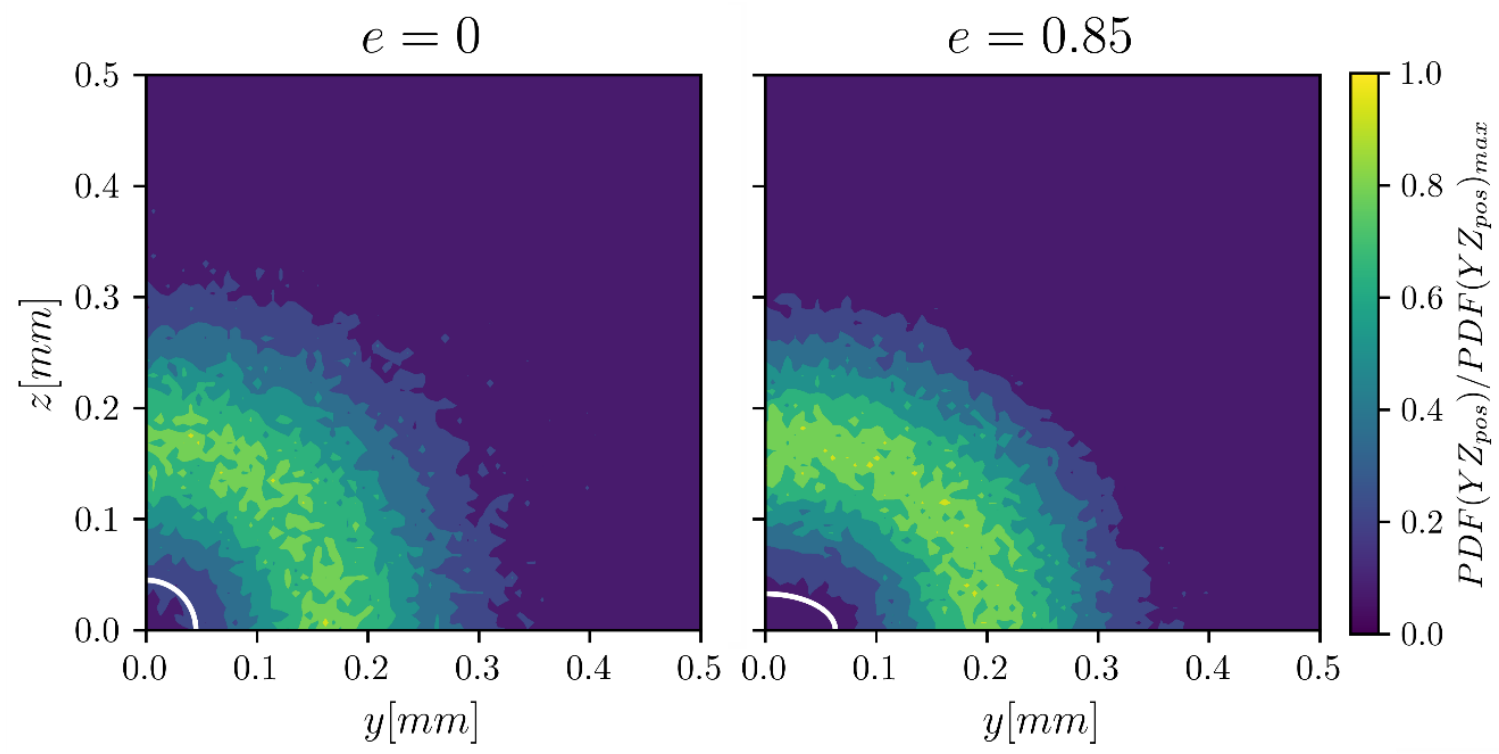

Figure 5: Bidimensional probability function of droplet position at YZ plane for left round jet and right elliptical jet.

\section{Conclusions and future outcomes}

DNS of primary atomization process have been performed in order to compare the influence of the nozzle cross-section on droplet generation. Even though the elliptical nozzle has slightly lower Reynolds number than the reference case, the total amount of droplets generated is higher. When it comes to the droplet size distribution, the number of fine droplets is higher for the elliptical jet, but the round jet presents a higher amount of big droplets. This leads to a different PDF of size distribution, the elliptical case showing a sharper. Therefore, even though the elliptical case is injecting less turbulence, the motion caused due to the asymmetry enhances the disintegration of the liquid core. Concerning the droplet position distribution, the droplet cloud generated is wider for the elliptical jet in the major axis plane and very similar in the minor axis plane. This behaviour is consistent with experimental and numerical studies done in elliptical jets and elliptical sprays, where the spray tends to get a circular shape as it develops.

The complete analysis of this simulations, as well as the turbulent field, will provide a detailed explanation of the presented effects. The main goal in future works will be centred on the study of the turbulence field and spectral behaviour of the elliptical case, incorporating more levels of eccentricity factor to get a trend on the influence of this parameter on the atomization pattern.

\section{Acknowledgments}

This research has been funded by the Spanish Ministerio de Ciencia e Innovación through the projects RTI2018-099706-B-100: "Estudio de la atomización primaria mediante simulaciones DNS y técnicas ópticas de muy alta resolución" and EQC2018-004605-P: "Estudio del proceso de inyección en atmosferas presurizadas". González-Montero L.A. is partially supported though the contract FPI - Subprograma 2 of the Universitat Politècnica de València (PAID01-18). 


\section{Nomenclature}

$\begin{array}{clll}u & \text { Velocity vector } & \text { Abbreviations } & \\ \rho & \text { Density } & \text { LES } & \text { Direct Numerical Simulation } \\ \mu & \text { Dynamic viscosity } & \text { VOF } & \text { Large Eddy Simulation } \\ \boldsymbol{D} & \text { Deformation tensor } & \text { CFD } & \text { Volume-Of-Fluid } \\ \sigma & \text { Surface tension } & \text { ECN } & \text { Computational Fluid Dynamics } \\ \kappa & \text { Liquid curvature } & \text { BC } & \text { Boundary Condition } \\ \partial_{s} & \text { Dirac function } & \text { PDF } & \text { Probability Density Function } \\ \boldsymbol{n} & \text { Liquid surface normal vector } & & \\ C & \text { Colour function } & & \\ e & \text { Eccentricity factor } & & \\ N_{d r o p} & \text { Number of drops } & & \\ d_{v} & \text { Volumetric diameter } & & \end{array}$

\section{References}

[1] F. Hussain and H. S. Husain, "Elliptic jets. Part 1. Characteristics of unexcited and excited jets," J. Fluid Mech., vol. 208, p. 257, Nov. 1989, doi: 10.1017/S0022112089002843.

[2] H. S. Husain and F. Hussain, "Elliptic jets. part 2. dynamics of coherent structures: Pairing," J. Fluid Mech., vol. 233, no. 439, pp. 439-482, 1991, doi: 10.1017/S0022112091000551.

[3] C.-M. Ho and E. Gutmark, "Vortex induction and mass entrainment in a small-aspect-ratio elliptic jet," J. Fluid Mech., vol. 179, p. 383, Jun. 1987, doi: 10.1017/S0022112087001587.

[4] C. Lee, I. Kim, K. Koo, J. Park, and Y. Lee, "Experimental study of the effects of nozzle hole geometry for a DI diesel engine," in ICLASS 2006, 2006.

[5] S. Yu et al., "Experimental study on the spray characteristics discharging from elliptical diesel nozzle at typical diesel engine conditions," Fuel, vol. 221, no. February, pp. 28-34, 2018, doi: 10.1016/j.fuel.2018.02.090.

[6] S. Yu et al., "Numerical investigation on effects of elliptical diesel nozzle on primary spray characteristics by large eddy simulation (les)," At. Sprays, vol. 28, no. 8, pp. 695-712, 2018, doi: 10.1615/AtomizSpr.2018026642.

[7] F. J. Salvador, J. M. Pastor, J. De la Morena, and E. C. Martínez-Miracle, "Computational study on the influence of nozzle eccentricity in spray formation by means of Eulerian $\Sigma-Y$ coupled simulations in diesel injection nozzles," Int. J. Multiph. Flow, vol. 129, p. 103338, 2020, doi: 10.1016/j.ijmultiphaseflow.2020.103338.

[8] A. J. Torregrosa, R. Payri, F. Javier Salvador, and M. Crialesi-Esposito, "Study of turbulence in atomizing liquid jets," Int. J. Multiph. Flow, vol. 129, p. 103328, 2020, doi: 10.1016/j.ijmultiphaseflow.2020.103328.

[9] ECN, "Engine Combustion Network," https://ecn.sandia.gov/diesel-spray-combustion/, 2010. www.sandia.gov/ecn/.

[10] S. Zaleski, G. Tryggvason, and R. Scardovelli, "ParisSimulator code," 2013. http://www.ida.upmc.fr/ zaleski/paris/index.html.

[11] G. Tryggvason, R. Scardovelli, and S. Zaleski, Direct numerical simulations of gas-liquid multiphase flows. Cambridge University Press, 2011.

[12] “OpenFOAM v3.0.0." 2015, [Online]. Available: https://openfoam.org/version/3-0-0/.

[13] F. J. Salvador, S. Ruiz, M. Crialesi-Esposito, and I. Blanquer, "Analysis on the Effects of Turbulent Inflow Conditions on Spray Primary Atomization in the Near-Field by Direct Numerical Simulation," Int. J. Multiph. Flow, vol. 102, pp. 49-63, 2018, doi: 10.1016/j.jmultiphaseflow.2018.01.019.

[14] R. Payri, F. J. Salvador, J. Gimeno, and M. Crialesi-Esposito, "Comparison of mapped and synthetic inflow boundary conditions in Direct Numerical Simulation of sprays," in ILASS Europe 2019, 29th Conference on Liquid Atomization and Spray Systems, 2019.

[15] A. L. Kastengren et al., "Measurements of droplet size in shear-driven atomization using ultrasmall angle x-ray scattering," Int. J. Multiph. Flow, vol. 92, pp. 131-139, 2017, doi: 10.1016/j.jmultiphaseflow.2017.03.005. 